\title{
Reliability Growth Testing Based on Dynamic Planning Methodology
}

\author{
Lu Yao, Jing Yang, Jianjun Yang \& Kai Chen \\ Department of Management Science, Naval University of Engineering \\ Wuhan 43003, China \\ E-mail: fzqy2046@163.com
}

\begin{abstract}
The reliability growth test is known as to stimulate malfunction, analyze the cause of the malfunction and improve of the design designedly, and carry out tests is to prove the effectiveness of improving measures. A large number of projects practice has proved that reliability growth test is an important and effective way in various stages of the work of the equipment reliability growth. This model search for the optimum target of reliability growth testing to make the cost using in whole reliability grow project in a low amount by considering the reliability growth testing as one stage of reliability grow project and basing on Dynamic Planning Methodology. Through the use of Dynamic Planning Methodology to arrange the target value at every stage of the work, so as to achieve the purpose of the minimum cost of the whole reliability project. After the target value is confirmed at the end of the various stages of the project, the target of growth of the reliability growth test as one of the projects is also determined accordingly.
\end{abstract}

Keywords: Reliability growth testing, Target of growth, Dynamic planning

\section{Instruction}

Target of growth of test is one of the important indexes that the test needs to establish, the reasonable growth targets will not only guarantee to achieve a satisfactory test results, but also shorten the test time and save the costs. (Mei, 2001, p.75).During determining the target of tests, we not only consider the cost savings of the test, but consider the reliability growth testing as one stage of reliability grow project in the premise of Technical Support.

\section{Build Cost Model}

This section takes the ultimate reliability level of the system as a state variable, the reliability of the completion of the Items (that is, the MTBF at the end of the work of the project) (Jin, 2002, p.119).as a decision-making program to establish optimal model. In order to determine the MTBF values at the end of the various stages of the project, so that under the premise of the reliability level of the product will meet the required the implementation of the decision-making make the total reliability cost the lowest. When the MTBF values are determined at the end of the various stages of the project, Target of growth as one project of the Reliability growth tests is determine accordingly. (Liu, 2000, p.63-65).

On the assumption that in the process of product development Products have the total number of $\mathrm{n}$ items which can increase the reliability of products, including reliability growth test is the $a$ project, the $\mathrm{n}$ work items have the time sequence, that is, after one of the projects is completed, the next work project starts, every project is on the basis of the preparatory work project. Clearly, the Completion Description of preparatory work will directly affect the costs inputs and Completion Description of the late work, thereby affecting the total cost of the development of products.

The following index model is a frequently-used cost account model in the reliability growth testing:

$$
C=A+B\left(\frac{M_{z}}{M_{q}}\right)^{\beta}
$$

Among them, $C$ express the development costs for product reliability; $A$ as the basic fixed costs; $B$ as the variable costs; $M_{q}$ as the original MTBF value of Product; $M_{z}$ as the MTBF value of the product is improved; $\beta$ as for the model parameters that can be obtained by fitting.

This article consider any the work of the project which caused reliability growth, can make use of the above-mentioned model, but the different project have different content itself, so their basic costs, variable costs and model parameters are not the same, the specific values can be accessed by the project's own related data. Thus, cost model of any $\mathrm{i}$ 
project as follows:

$$
C_{i}=A_{i}+B_{i}\left(\frac{M_{i}}{M_{i-1}}\right)^{\beta_{i}}
$$

Among them, $M_{i-1}$ as at the end of the preparatory work project, the MTBF value at the beginning of the work item; $M_{i}$ as the MTBF value at the end of the work item.

Correspondingly, the total reliability project cost is sum of the reliability costs of each sub-project: $C=\sum_{i=1}^{n} C_{i}$

\section{Build Optimization Models}

On the assumption that the initial value of the MTBF of Products is $M_{0}$, according to the order we carried out project work in order to increase the reliability of products, so that the final value of the MTBF is $M$, in which the reliability growth test is the $a(1 \leq a \leq n)$ projects. In this paper, the optimal strategy is as follows:

By adopting the ultimate reliability level of system as a state variable, the completion of the reliability of the projects (that is, the value of MTBF at the end of the work of the project). (Gan, 1999, p.69).as a decision-making program to establish optimal model. In order to determine the MTBF values at the end of the various stages of the project, so that under the premise of the reliability level of the product will meet the required the implementation of the decision-making make the total reliability cost the lowest.

Specific optimization model is as follows:

$$
\begin{aligned}
& \operatorname{Min} C(M)=\sum_{i=1}^{n} A_{i}+B_{i}\left(M_{i} / M_{i-1}\right)^{\beta_{i}} \quad \cdots \cdots \\
& \text { s.t. } M_{0} \leq M_{1} \leq \cdots \leq M_{n-1} \leq M_{n}=M
\end{aligned}
$$

\section{The Research of Model Solution}

Since variables of the model can not be separated, so it can not be directly solved by dynamic programming methods. In this paper, variables are separated through introducing magnification factor $k_{i}$ to the model. The definition of magnification factor $k_{i}$ is enlarging multiples of the value of MTBF of product compared with the early start of the project after the $i$ work item. Substitute it into the optimization model formula can be turned into:

$$
\begin{gathered}
\operatorname{Min} C\left(k_{i}\right)=\sum_{i=1}^{n} A_{i}+B_{i}\left(k_{i}\right)^{\beta_{i}} \\
\text { s.t. }\left\{\begin{array}{l}
k_{i}=M_{i} / M_{i-1} \\
1 \leq k_{i} ; k_{1} * k_{2} \cdots k_{n-1} * k_{n}=\frac{M}{M_{0}}
\end{array}\right.
\end{gathered}
$$

For the above-mentioned formula, it can be solved by the dynamic programming order method.

Set up: When a work item corresponds to a phase, that is, the number of phase sequence is $i$; the state variable is defined as the multiples which has been enlarged of the value of MTBF of the product before stage $i$,decision-making variables $k_{i}$ expresses enlarge multiples of the value of MTBF of Products from stage $i-1$ to stage $i$

The state transition equation is as follows: $s_{i+1}=s_{i} * k_{i}$

Set of admissible decisions is $D_{i}\left(s_{i}\right)\left\{1 \leq k_{i} \leq s_{i+1}\right\}$

Index function at various stages is the cost inputs of each working item: $v_{i}\left(k_{i}\right)=A_{i}+B_{i} k_{i}^{\beta_{i}}$

The optimal function values expresses the spent minimal cost by magnifying the value of MTBF of the product in the working item $i$ by $k_{i}$ times, thus the order recursion relations of the dynamic programming can be written into:

$$
\begin{aligned}
& f_{i}\left(s_{i+1}\right)=\operatorname{Min}\left\{v_{i}\left(s_{i+1}, k_{i}\right)+f_{i-1}\left(s_{i}\right)\right\} \\
& i=1,2, \cdots, n
\end{aligned}
$$

Among them, at the time $s_{0}=1$, it means that the cost inputs of product is zero when the value of MTBF of product is not increased, that is, the boundary conditions are as follows: $f_{0}\left(s_{1}\right)=0$

According to the above methods, we can get the magnification factor $k_{i}$ of each work item; the value of MTBF at the 
end of the work item is $M_{i}=M_{i-1} * k_{i}$ among $i \geq 1$ and $M_{0}$ is known.

Accordingly, the growth objectives of the $a$ Reliability growth testing can be established: $M_{a}=M_{a-1} * k_{a}$.

\section{Examples}

Known in the process of some product development, the initial value of MTBF of product is $M_{0}=100$ hours, now we plan to improve of the value of MTBF which will not be less than $M_{3}=900$ hours through three reliability work item, the three projects carried out by time sequence, in which reliability growth testing is the second work item.

The cost of Three working project and the relationship model of reliability are as follows:

$$
\begin{aligned}
& C_{1}=350+300\left(\frac{M_{z}}{M_{q}}\right)^{2}, \\
& C_{2}=400+280\left(\frac{M_{z}}{M_{q}}\right)^{2.5}, \\
& C_{3}=500+350\left(\frac{M_{z}}{M_{q}}\right)^{3}
\end{aligned}
$$

$M_{z}$ is the value of MTBF at the end of the work item;

$M_{q}$ is the value of MTBF at the beginning of the work item.

Requested under the premise of meeting the requirements the final value of MTBF of Products, we arrange for the three projects reasonably, making the total cost of reliability the lowest.

By substituting Known conditions into the above optimization model we get:

$$
\begin{aligned}
& \operatorname{Min} C(k)=1250+500 k_{1}^{2}+300 k_{2}^{2.5}+500 k_{3}^{3} \\
& \text { s.t. }\left\{\begin{array}{l}
1 \leq k_{i} ; \\
k_{1} * k_{2} * k_{3}=9
\end{array}\right.
\end{aligned}
$$

in that $k_{i}=M_{i} / M_{i-1}, i=1,2,3$.

Three work items can be taken as a three-stage decision-making problem set state variables are $s_{1}, s_{2}, s_{3}, s_{4}$,

$s_{i}$ expresses the multiples which has been expanded compared with the values of MTBF of the Product at early stages $i, s_{1}=1$

Adopting $k_{1}, k_{2}, k_{3}$ as the decision variable;

Index function at various stages is:

$$
\begin{aligned}
& v_{1}\left(k_{1}\right)=1250+500 k_{1}^{2}, \\
& v_{2}\left(k_{2}\right)=500 k_{2}^{2.5}, \\
& v_{3}\left(k_{3}\right)=500 k_{3}^{3}
\end{aligned}
$$

The state transition equation is as follows:

$$
\begin{aligned}
& \mathrm{s}_{1}=1, \\
& s_{2}=s_{1} * k_{1}, \\
& s_{3}=s_{2} * k_{2}, \\
& s_{4}=s_{3} * k_{3}=9 \\
& k_{1}=\mathrm{s}_{2}, \quad 1 \leq \mathrm{k}_{2} \leq \mathrm{s}_{3}, \quad 1 \leq \mathrm{k}_{3} \leq s_{4}
\end{aligned}
$$

Supposed the optimal value function $f_{i}\left(s_{i+1}\right)$ expresses the minimum value of the cost of reliability from the first stage to the $i$ stage, so the order recursion relations are: 


$$
\left\{\begin{array}{l}
f_{i}\left(s_{i+1}\right)=\operatorname{Min}\left\{v_{i}\left(s_{i+1}, k_{i}\right)+f_{i-1}\left(s_{i}\right)\right\} \quad \ldots \ldots \\
i=1,2,3 \\
f_{0}\left(s_{1}\right)=0
\end{array}\right.
$$

By substituting related conditions into the recursive relationship respectively and using differentiation we get:

$$
k_{1}=2.695, k_{2}=2.0781, k_{3}=1.607
$$

Then By substituting the initial value of MTBF of products into $f_{i}\left(s_{i+1}\right)$,we can get the optimized reliability objectives of the work item are as follows:

$$
M_{1}=235, M_{2}=560, M_{3}=900
$$

So when we carry out the reliability growth testing, growth target of product may be set as its value of MTBF is 560 hours, the optimized purpose can be achieved.

\section{Summary}

It should be noted that:

When the model solution obtained is $k_{i}=1$, purely from the economic perspective it means that the reliability of the work item is worthwhile and it should not be carried out.

However, in engineering practice, it does not mean that the work item can be omitted, we should arrange the work item by the required minimum input conditions of project, and then by substituting the cost and its growth of reliability which is regarded as a certainty value into the original model and resolving, we can get the optimized growth targets of other work item.

\section{References}

Gan, Ying'ai. (1999). Operational Research. (3rd ed.). Qing Hua University Press.

Jin, Bihui. (2002). System Reliability Engineering. National Defence Industry Press.

Liu, Xiaodong and Zhang, Hengxi. (2000). Flight System Reliability Develop Forecast Model Cost Research. Quantity and Technology Economic Research, 12, p.63-65.

Mei, Wenhua. (2001). Reliability Growth Testing. National Defence Industry Press. 